\title{
Charging an Electronic Gadget using Piezoelectricity
}

\author{
J. John Livingston* and M. Hemalatha \\ School of Computing, SASTRA University, Thanjavur, Tamil Nadu, India; \\ Johnlivingston03@gmail.com, hemalatha@it.sastra.edu
}

\begin{abstract}
The method of generating electrical voltage for charging an electronic gadget from the piezoelectric sensor that is fixed to the sole of the footwear is illustrated in this paper.
\end{abstract}

Keywords: Energy Harvesting, Piezoelectricity, Piezoelectric Sensor, Portable Electric Power

\section{Introduction}

With the introduction of many handheld portable electronic gadgets, energy harvesting has become one of the fascinating subjects of interest to provide portable electrical power. The commonly used sources are: solar power, wind energy and piezoelectricity. This study is focussed on Piezoelectricity as it depends on the mechanical pressure or strains to obtain electrical energy, while the other sources are not reliable at all times.

\section{Existing Mechanical Energy Harvesters}

Some vibration based energy generators that converts mechanical energy to electrical energy that have been successfully developed are Electromagnetic ${ }^{1}$, Electrostatic ${ }^{2}$, Piezoelectric generators ${ }^{3,4}$.

\subsection{Electromagnetic Generator}

Whenever a change in the magnetic flux linked with the coil occurs, an electromagnetic force (emf) is generated across the coil in an electromagnetic generator. The relationship between the emf and the displacement $(z)^{5}$ is

$$
\text { emf }=K \frac{d z}{d t}
$$

where, the constant $\mathrm{K}$ is determined by number of turns of the coil, length of the coil, area of the coil and magnetic field intensity.

\subsection{Electrostatic Generator}

It consists of a capacitor whose capacitance (C) changes as a function of displacement $(Z)$. The equation for the electric current flowing in an electrostatic generator ${ }^{5}$ is given by

$$
I=V_{0} \frac{d C(z)}{d t}+\frac{C(z) d V}{d t}
$$

where, V represents the voltage that is developed across the capacitor when the capacitor is fully charged, $\mathrm{V}_{0}$ represents the initial value of the voltage across the capacitor. $\mathrm{V}_{0} \frac{d C(z)}{d t}$ represents electromechanical coupling $\frac{C(z) d V}{d t}$ and represents the relationship between the current and the capacitance. When the capacitor is charged with $\mathrm{V}_{0}$ initially then the electromechanical coupling will be high. The polarisation of the capacitor can be achieved by using piezoelectric elements as done in this paper ${ }^{5}$.

\subsection{Piezoelectric Generator}

There are two effects in piezoelectricity: direct piezoelectric effect and converse piezoelectric effect. Direct piezoelectric

${ }^{*}$ Author for correspondence 
effect is the generation of electric charge when mechanical strain is applied on piezoelectric materials; whereas the converse effect is defined as the deformation in the crystal caused, when an electrical voltage is applied to the piezoelectric materials. Direct piezoelectric effect is employed in manufacturing sensors and converse piezoelectric effect is employed in manufacturing actuators. The piezoelectric materials are of natural and also of artificially made. Quartz is a natural piezoelectric material while Lead Zirconate Titanate (PZT) is an artificial piezoelectric material.

The following equation ${ }^{6}$ describes piezoelectric effect (3) and its converse effect (4).

where, $\mathrm{D}$ denotes electric displacement vector, $\mathrm{T}$

$$
\begin{aligned}
& D=d . T+T . E \\
& S=s E . T+d t . E
\end{aligned}
$$

denotes stress vector, $E$ T denotes dielectric permittivity at constant mechanical stress, $s E$ denotes the matrix for compliance coefficients at constant electric field, $S$ denotes strain vector, $\mathrm{d}$ denotes piezoelectric constant matrix, E denotes electric field vector.

In piezoelectric effect, charge density is developed on its surface when external pressure is applied ${ }^{5}$.

where, $\mathrm{D}$ is the charge density, $\mathrm{Q}$ is the electric charge

$$
D=\frac{Q}{A}
$$

that is accumulated on the surface, $\mathrm{A}$ is the area of the conductive electrode.

Table 1 shows the comparison of energy storage density between the three generators ${ }^{5}$.

The table 1 also shows that piezoelectric has more energy storage density when compared with the other techniques. PZT piezoelectric sensor can be used for small scale energy harvesting because of its high energy storage density ${ }^{7}$.

\section{System Description}

Energy harvesting involves harvesting electrical power and storing of the harvested power. The reason for

Table 1. Energy storage density comparison

\begin{tabular}{lc}
\hline $\begin{array}{l}\text { Energy harvester working } \\
\text { principle }\end{array}$ & $\begin{array}{c}\text { Energy storage density } \\
\left(\mathrm{mJ} / \mathrm{cm}^{3}\right)\end{array}$ \\
\hline Electromagnetic & 24.8 \\
Electrostatic & 4 \\
Piezoelectric & 35.4 \\
\hline
\end{tabular}

choosing piezoelectricity is because of its higher energy storage density as it has been justified earlier. In piezoelectric energy harvesting, piezoelectric sensor is used as a harvesting element and the storage element is a battery.

\subsection{Piezoelectric Sensor}

It converts the mechanical stress to electrical voltage. When the mechanical stress is applied to the sensor, electrical charge is accumulated on the crystal surface that can be extracted using a wire. In order to get maximum output from the sensor, it has to be set in its self-resonant frequency range. Piezoelectric sensor can be considered as an electrical equivalent of combination of resistance $\mathrm{R}$, capacitance $\mathrm{C}$ and an alternating current source I connected in parallel as shown in Figure $1^{6}$.

The resonant frequency can be found by using Helmholtz equation ${ }^{6}$ that is given below:

$$
f=\frac{c \frac{\sqrt{4 a^{2}}}{d^{2} h(t+k a)}}{2 \pi}
$$

where, $f$ represents the resonant frequency, $c$ represents the velocity of the energy wave, 'a' represents the radius of ceramic diaphragm, 'd' represents the diameter of support, ' $\mathrm{t}$ ' represents the thickness of support, ' $k$ ' represents the material constant.

The sensor used in this work is made of piezoceramic material Lead Zirconate Titanate (PZT). It is circular in shape which fits comfortably into the sole of the footwear and it is commercially available at low cost. Individual sensor when tested using Cathode Ray Oscilloscope (CRO) generates a variable voltage of 4-6V (Figure 2).

In this work, four piezoelectric sensors are connected in parallel that are fitted in to the sole of the footwear. During jogging and jumping, mechanical stress that is

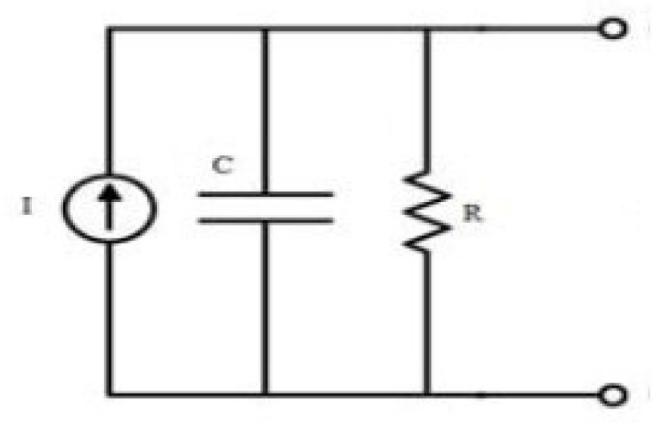

Figure 1. Electrical model of piezoelectric sensor. 


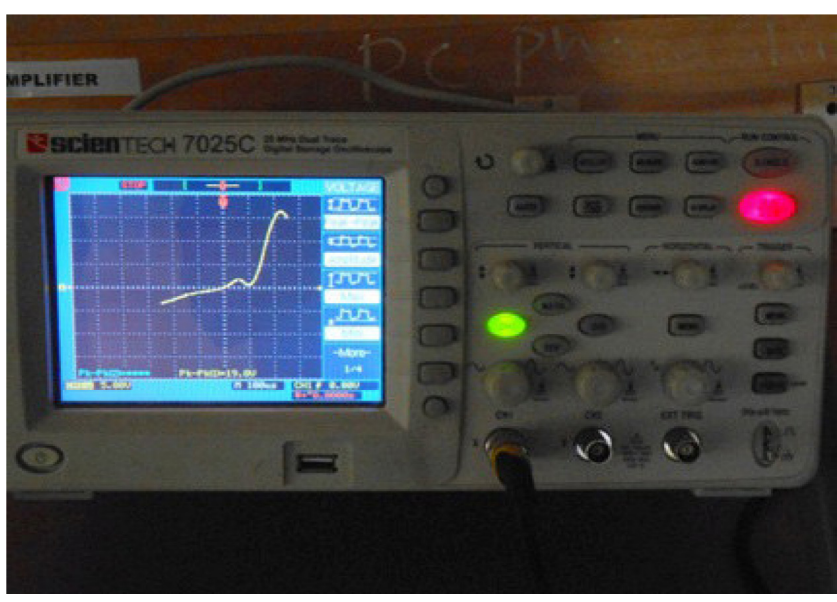

Figure 2. Piezoelectric sensor tested in CRO.

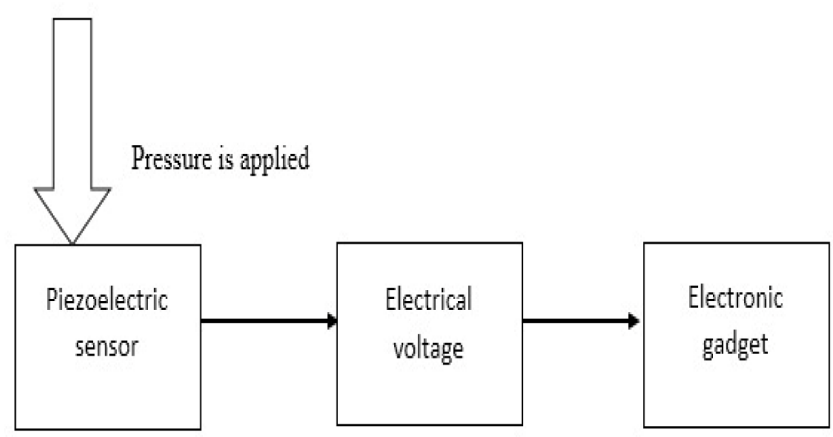

Figure 3. Piezoelectric energy harvesting process.

applied on the sole of the footwear is converted to electrical voltage which is given to the electronic gadget (Figure 3). A human while walking exerts about $30 \mathrm{~W}$ of power on the ground and theoretically out of which $100 \mathrm{~mW}$ of electrical power is possible to obtain without disturbing the comfort of the person's walk ${ }^{9}$.

\subsection{Positioning of Piezoelectric Sensor}

Piezoelectric sensors has to be positioned in two main parts of the sole of the shoe where the maximum pressure is applied (Figure 4).

Single sensor is capable of generating $3-5 \mathrm{~V}$ on application of pressure consistently, in this work four sensors are connected in parallel in order to increase the probability of getting maximum output.

Piezopolymeric materials are more advantageous to use than piezoceramic materials in case of sensor application, because polymeric films can be easily fabricated to different shapes. Even then piezoceramic sensor has been used in this work because it is commercially available at low cost (Figure 5).

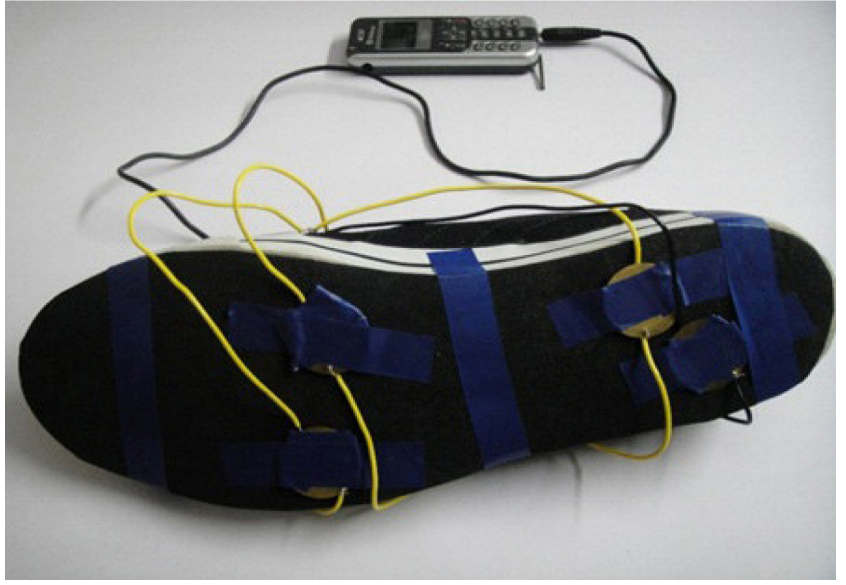

Figure 4. Positioning of piezoelectric sensor.

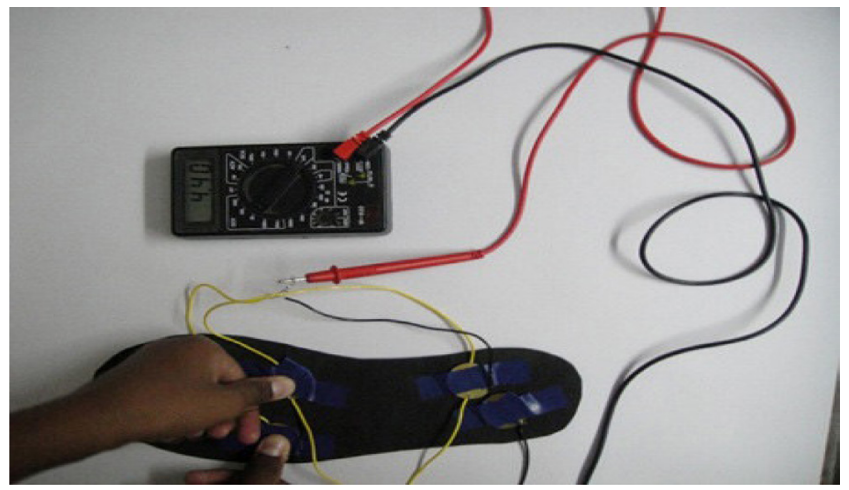

Figure 5. Testing of sensor using multimeter.

\subsection{Storage device}

The load that is used for storing the harvested electrical energy is mobile phone battery. Many mobile phones use a battery which is made of the combination of chemicals Lithium-ion "Li-ion" that needs a DC voltage of $3.6 \mathrm{~V}$ for charging. For power storage it is better to use rechargeable battery than a super capacitor as rechargeable battery has high energy stored per unit weight and a slower discharge response $\mathrm{e}^{10}$.

\section{Experimental Results}

The necessary voltage required for charging a mobile phone battery is successfully generated and the output is shown in the picture. The output current that is generated from the piezoelectric sensor may be less, which may increase the time taken for charging a battery. But it can be used for charging an electronic device battery for emergency purpose where there is no direct source of electricity. This can be used as an efficient source for portable electric power 


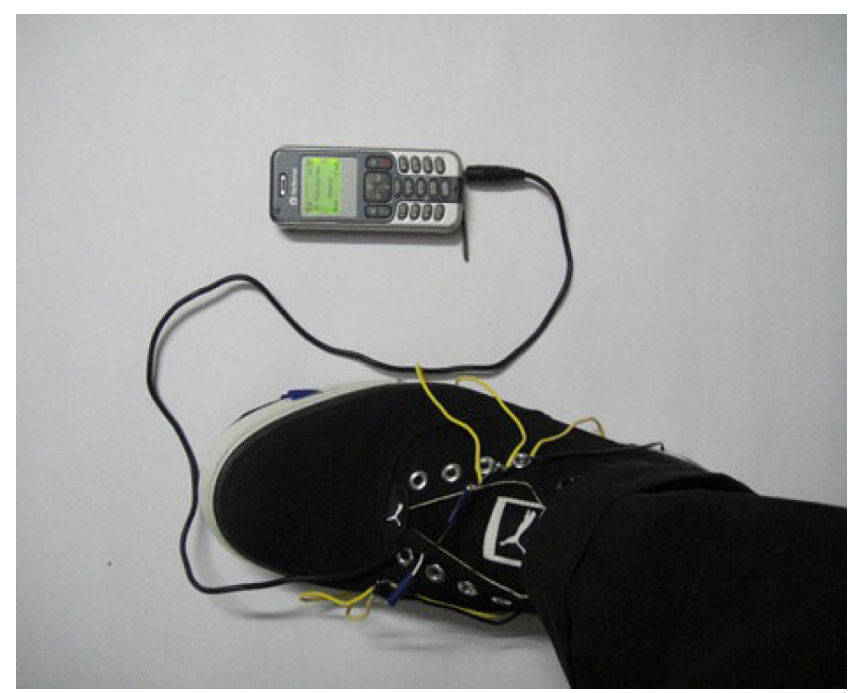

Figure 6. Experimental Output.

for portable devices. This work is a low cost approach to demonstrate the application of piezoelectric sensor to meet the need for portable electric power (Figure 6).

\section{Future Scope}

The analysis of the voltage stored in the battery with respect to the force applied can be done and the efficiency of this work can be calculated.

Efficient storage of the generated voltage can be achieved by using the circuit shown in Figure 7.

An array of piezosensors can be connected in series to get larger output and can be used for higher load applications. A rectifier-free piezoelectric energy harvesting circuit can be used for charging efficiently8. Piezoelectric energy harvesting can be used as a cleanest form of alternate energy source in future. This work is an example illustrating one of its applications. It can also be used as an energy source for wearable electronics.

\section{References}

1. El-hami M, Glynne-Jones P, White M, Hill M, Beeby S, James E, Brown D, Ross N. Design and fabrication of a new

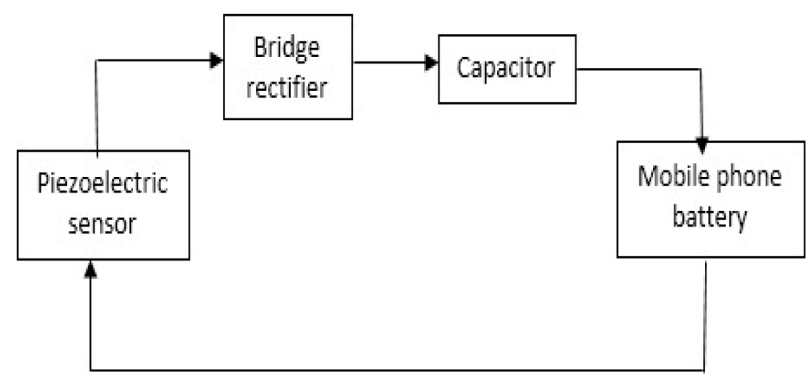

Figure 7. Circuit for efficient energy harvesting.

vibration-based electromechanical power generator. Sens Actuators A, Phys. 2001; 92(1-3):335-42.

2. Miyazaki M, Tanaka H, Ono G, Nagano T, Ohkubo N, Kawahara T, Yano K. Electric-energy generation using variable capacitive resonator for power-free LSI. Proc ISLPED; 2003. p. 193-98.

3. Keawboonchuay C, Engel TG. Maximum power generation in a piezoelectric pulse generator. IEEE Trans Plasma Sci. 2003; 31(1):123-28.

4. Yang J, Chen Z, Hu Y. An exact analysis of a rectangular plate piezoelectric generator. IEEE Trans Ultrason, Ferroelectr Freq Control. 2007; 54(1):190-95.

5. Rocha JG, Gonçalves LM, Rocha PF, Silva MP, LancerosMéndez S. Energy harvesting from piezoelectric materials fully integrated in footwear. IEEE Trans Ind Electron. 2010; 57(3):813-19.

6. PisharodyHarikrishnan G. An optimal design for piezoelectric energy harvesting system. IEEE PES Innovative Smart Grid Technologies- India; 2007.

7. Turner J, Ahmed MN, Ha DS, Mattavelli P. A new approach to the wide bandwidth of piezoelectric transducers for vibration energy harvesting. IEEE. 2010; 1064-67.

8. Kwon D, Rincon-Mora GA. A Rectifier-Free Piezoelectric Energy Harvester Circuit.IEEE. 2009; 1085-88.

9. Han D, Kaajakari V. Microstructured polymer for shoe power generation. Transducers, Denver, CO, USA; 2009.

10. Ali WG, Nagib G. Design Considerations for Piezoelectric Energy Harvesting Systems. 2012 International Conference on Engineering and Technology (ICET); 2012 Oct 10-11. Cairo. IEEE. p. 1-6. 